\title{
Cannabis Use among People Entering Drug Treatment in Europe: A Growing Phenomenon?
}

\author{
Linda Montanari $^{\mathrm{a}}$ Bruno Guarita $^{\mathrm{a}} \quad$ Jane Mounteney $^{\mathrm{a}} \quad$ Nina Zipfel ${ }^{\mathrm{b}}$ \\ Roland Simon ${ }^{a}$ \\ ${ }^{a}$ European Monitoring Centre for Drugs and Drug Addiction (EMCDDA), Lisboa, Portugal; ${ }^{\mathrm{b}}$ Maastricht University, \\ Maastricht, Netherlands
}

\section{Keywords}

Cannabis - Treatment entries · Trends · Europe · Joinpoint regression

\begin{abstract}
This paper explores European and national trends in specialised drug treatment entry for cannabis-related problems. The analysis is based on data for the years 2003-2014 from 22 European countries. Between 2003 and 2014, the overall number and proportion of primary cannabis-related firsttime entrants increased significantly. A joinpoint regression analysis indicates that the overall increase of cannabis treatment entries is continuous, although country-related differences are observed. Possible explanations for the increase and different time trends are discussed including an increase in cannabis prevalence and cannabis-related problems, changes in risk perception, increases in cannabis potency, changes in referral practices and increased availability and accessibility of treatment services.

(c) 2017 S. Karger AG, Basel
\end{abstract}

\section{Introduction}

Cannabis is the most widely consumed illicit drug in Europe and worldwide [1]. In 2014, an estimated 83 million (25\%) European adults aged 15-64 had used cannabis at least once in their lifetime, with higher lifetime prevalence levels (34\%) among young adults (15-34) [2, $3]$. Estimates indicate that around $1 \%$ of European adults are daily or almost daily cannabis users [2], having used the drug on 20 days or more in the last month. Regular and long-term cannabis use is associated with increased risk of health problems including dependence [4]. Recent estimates suggest that cannabis dependence may occur in around $11 \%$ of lifetime cannabis users with an increased risk of dependence among those who initiated cannabis use in adolescence and among daily users [5]. Cannabis dependence is a disorder primarily experienced by young adults, and the health burden is reportedly greater in higher income countries [6]. Although not correlated with increased mortality, cannabis dependence may lead

\section{KARGER}

(c) 2017 S. Karger AG, Basel

E-Mail karger@karger.com

www.karger.com/ear
Linda Montanari

European Monitoring Centre for Drugs and Drug Addiction (EMCDDA)

Praça Europa, 1, PT-1249-289 Lisboa (Portugal)

E-Mail Linda.Montanari@emcdda.europa.eu 
to negative health outcomes, such as cognitive impairment, mental disorders, cardiovascular disease, chronic obstructive pulmonary disease and respiratory and other cancers [5]. Because of high rates of cannabis use, cannabis-related problems may concern a large number of people and may be associated with entry into specialised drug treatment [7].

\section{Aim}

This paper explores European and national trends in first-time treatment entrants for primary cannabis-related problems during the period 2003-2014 in Europe. The outcome of the analysis may have important practical implications for drug treatment policy development, planning and the optimal organisation of national and local drug treatment systems.

\section{Methods and Measures}

National data on specialised drug treatment entries is provided annually to the European Monitoring Centre for Drugs and Drug Addiction (EMCDDA) $[8,9]$ by 30 European countries (28 EU Member States, Norway and Turkey). This constitutes a common European data-collection method and monitoring system (called "treatment demand indicator" or TDI). This system collects information on people entering treatment, including information on the number and profile of individuals entering specialised drug treatment in Europe based on a standard protocol and following detailed methodological specifications and guidelines [8]. In this paper, the term "treatment demand" is used specifically to refer to treatment entries. The collected variables include socio-demographic characteristics, patterns of drug use and use of services. Specialised drug treatment may be provided in outpatient or inpatient centres, low threshold agencies, general practitioners or treatment units in prison. This information is collected at treatment centres by the professionals and forwarded to regional and national institutions, which then report data to the EMCDDA in an aggregate form. Clients are given a report only once annually, even if they may have re-entered treatment during the same year. The treatment centres included in the current analysis are specialised treatment units treating drug addiction (outpatient and inpatient treatment units, low threshold agencies, general practitioners and other types of treatment centres [8]). Based on available data, most cannabis clients are treated in outpatient units and only a minority are treated in inpatient or other types of units [10]. They are categorised on the basis of the primary drug used, which is the drug reported to be causing the client the most problems at the start of treatment. The classification is based on clients' statements when requesting treatment and (or) on the diagnosis made by a treatment professional.

This analysis focuses on clients entering specialised drug treatment for primary cannabis-related problems for the first time in their life in Europe between 2003 and 2014. Countries were included if data was available for at least 9 of the 11 years analysed (22 out of 30 countries - Europe-22). These were Bulgaria, Cyprus,
Czech Republic, Finland, France, Germany, Greece, Hungary, Ireland, Italy, Latvia, Lithuania, Luxembourg, Malta, Portugal, Romania, Slovakia, Slovenia, Spain, the Netherlands, United Kingdom, and Turkey. Countries with more than 2 missing data points were excluded (Austria, Belgium, Croatia, Denmark, Estonia, Norway, and Poland), as were Sweden, where substantial changes occurred in the treatment-monitoring system during the study period. This would have made the comparison between years impossible. For Luxembourg, the number of first-time clients was small (19); therefore, data on all clients entering treatment during the year was used (i.e., first-time clients + clients who have re-entered drug treatment during the reporting year). For the EU-22 sample with 9 to -11 data points, missing data points were imputed by the most recent neighbouring data point. This was necessary for France (2003, 2004); Italy (2003); Lithuania (2003); Malta (2007, 2008); Portugal (2003, 2004); Spain (2003, 2014), the United Kingdom (2012), and Turkey (2003).

Trends were analysed using joinpoint regression [11-13], which describes the direction and the magnitude of trends fitting linear segments over the period of analysis. This exploratory method estimates the points in time when linear trend changes occur ("joinpoints"), without prior theoretical hypothesis, finding the best-fit line through the available data of a specified period of time. Joined linear segments are fitted and each joinpoint indicates a change in trend. The number of joinpoints is determined by a permutation test, which establishes the null distribution of a statistical test. Thus, the joinpoint indicates a statistically significant change in trend. The minimum and maximum number of joinpoints are set in advance, where 0 joinpoints are indicative for a one-line segment. If one joinpoint shows to be significant, more joinpoints (up to 2 ) are added to the model. Statistical significance was set at $p<0.05$ for the joinpoint segments. The annual percent change (APC) tested the hypothesis whether it is equal to zero, and tested the statistical significance of the model. The APC assumed that rates that change at a constant percentage every year change linearly on a log scale. A value in either APC $<-1$ is considered a downward trend, whereas an $\mathrm{APC}>1$ indicates an upward trend. A trend is considered stable if $-1 \leq \mathrm{APC} \leq 1$. In order to identify and compare trends over the same period when there was one or more joinpoints, the average APC (AAPC) was estimated as a geometric weighted average of the APCs of the segments adjacent to the joinpoints, with the weights equal to the lengths of each segment over the interval. The software used in the analysis is the Joinpoint Regression Analysis Software [11].

\section{Results}

\section{EU Prevalence and Trends}

In 2014, 400,125 clients entered specialised drug treatment in the 22 European countries included in this analysis (EU-22) and cannabis was the main drug used as reported by 116,673 clients or $29 \%$ of all drugs clients. In the same year, a total of 67,444 first-time cannabis clients were reported as entering treatment in these 22 countries, representing $46 \%$ of all new drug clients $[2,3]$. The majority of these new clients were from the United Kingdom $(15,895)$, Germany $(14,458)$, Spain $(12,912)$, France 
$(6,897)$, and Italy (5,267; Table 1$)$. Considerable national variation exists in the number of first-time cannabis entrants, ranging from 27 clients in Bulgaria to 15,895 clients in the United Kingdom. Large country differences were also evident in the relative proportion of cannabis users entering treatment out of all drugs clients, ranging from 8\% in Bulgaria to 77\% in Cyprus and France [2].

Over time, the number of first-time cannabis treatment entrants in EU-22 significantly increased from 32,178 clients in 2003 to 67,444 in 2014 (AAPC $=6.11 \%$ per year; Table 1). The increase was continuous, or without statistically significant interruptions of the trends, during this period (Fig. 1). The number of daily cannabis users entering treatment also increased from 10,353 in 2003 to 28030 in 2014 [14].

The cannabis clients entering treatment for the first time in 2014 spent 2 years longer outside treatment than their equivalents in 2003, and were on average 2 years older ( 25 vs. 23 ) when they entered treatment, although starting cannabis use at the same age [16].

The increases seen for cannabis do not appear to be due to an overall increase in the numbers of clients entering specialised treatment in Europe for primary illicit substances, as also the proportion of primary cannabis clients among all new drug clients increased notably during the same period, rising from $29 \%$ of all new entrants in 2003 to $46 \%$ in 2014 (Table 1) [2, 15].

\section{National Trends}

Considerable national variations were observed in time trends for a number of cannabis clients entering specialised drug treatment for the first time between 2003 and 2014. Increasing trends were observed in 16 countries (Cyprus, France, Germany, Greece, Ireland, Latvia, Lithuania, Luxembourg, Malta, Portugal, Romania, Slovakia, Spain, the Netherlands, United Kingdom, and Turkey) and decreasing trends in 6 countries (Bulgaria, Czech Republic, Finland, Hungary, Italy, and Slovenia).

For the 14 countries with statistically significant increasing trends ${ }^{1}$, the AAPC was $>20$ in 3 countries (Cyprus, Lithuania, Romania); it was between 10 and 19 in 5 countries (France, Greece, Luxembourg, Spain, and the United Kingdom) and between $>1$ and 10 in 6 countries (Germany, Ireland, Latvia, Portugal, Slovakia, and the Netherlands). For the 4 countries with statistically significant decreasing trends ${ }^{2}$, the AAPC ranged between -2.7 and -12.5 .

\footnotetext{
${ }^{1}$ Non-statistically significant increases were reported in Malta and Turkey. ${ }^{2}$ Non-statistically significant decreases were reported in Bulgaria and Slovenia.
}

Cannabis Trends Treatment Europe
Joinpoint regression analysis revealed that 14 countries reported unbroken trends, either increasing or decreasing, for the period 2003-2014 and 8 countries interrupted trends with identified joinpoints (Czech Republic, France, Ireland, Romania, Slovenia, Spain, the Netherlands, and the United Kingdom; Table 1).

The countries with interrupted trends (Fig. 2) appear to cluster into 4 patterns: (a) the countries with an initial decrease followed by an increase (Ireland, Spain); (b) the countries with 2 periods of increase, where one phase was more accentuated than the other (France, the United Kingdom, and Romania); (c) the countries with a decrease followed by a stable phase (Slovenia, Czech Republic); and (d) one country (the Netherlands) with an initial increase followed by a decrease.

\section{Discussion}

This analysis highlights a doubling in the number of individuals entering specialised drug treatment for cannabis-related problems for the first time in EU-22 between 2003 and 2014. The overall increasing trend is confirmed by the joinpoint regression analysis showing an increasing trend at an AAPC of $6.11 \%$ of the absolute number of first-time clients entering treatment. Similar increases have not been reported for other illicit drugs, and cannabis problems appear to be responsible for an increasing percentage of all new drug treatment demands $[2,13,16]$. Considerable national variations were observed in time trends for a number of cannabis clients entering treatment for the first time.

A range of possible factors, including prevalence and trends; drug potency and harms; treatment need and provision; policy developments; and data-collection practices may have influenced the observed overall increase in the number of cannabis treatment demands in Europe as well as the varying national trends. In the next section, some potential explanatory factors are highlighted; although a detailed review of country-level analysis is not conducted, it is hoped that results provide a platform for future research and investigation in this area.

From the beginning of the 1990s and into the early 2000s, many European countries saw cannabis consumption rates increasing, even though only a limited number of countries have sufficient survey data to enable the possibility of performing a statistical analysis of medium- and long-term trends [17, 18]: Nevertheless, because of the large number of people involved in cannabis consumption, even a small increase in cannabis 


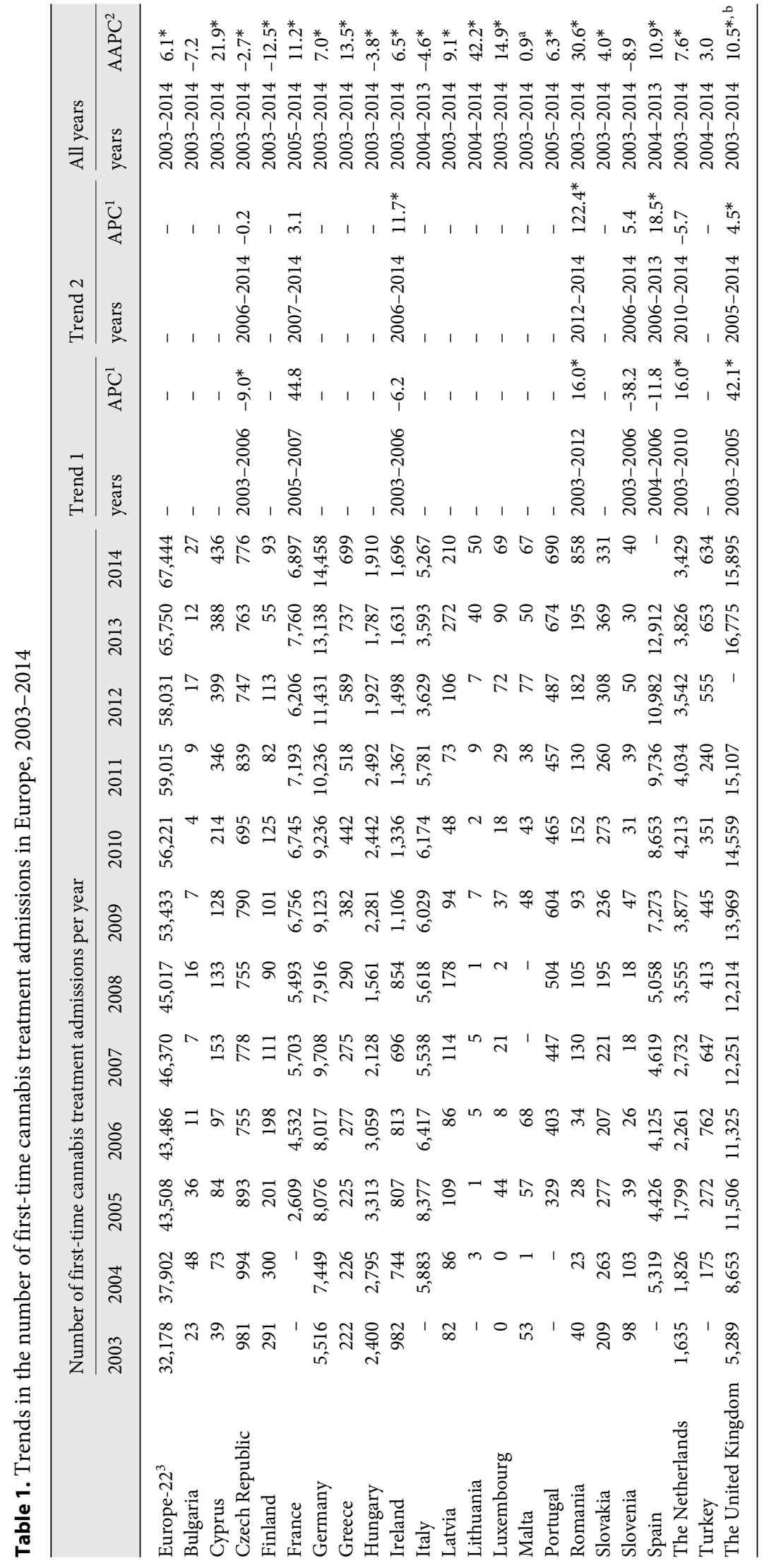

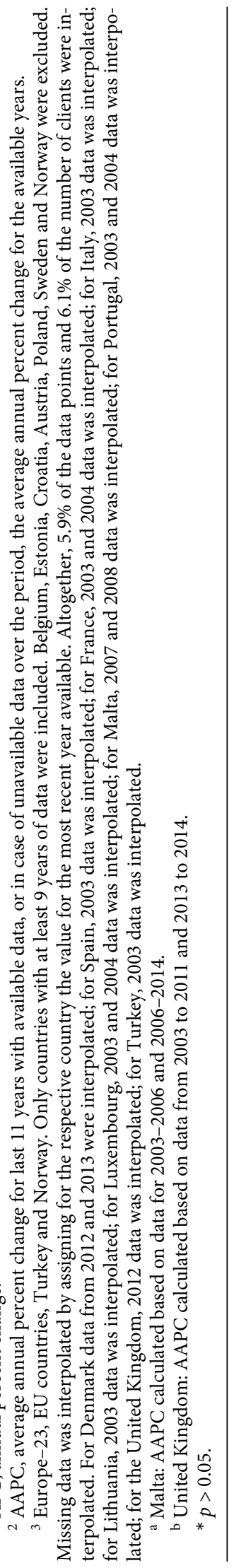




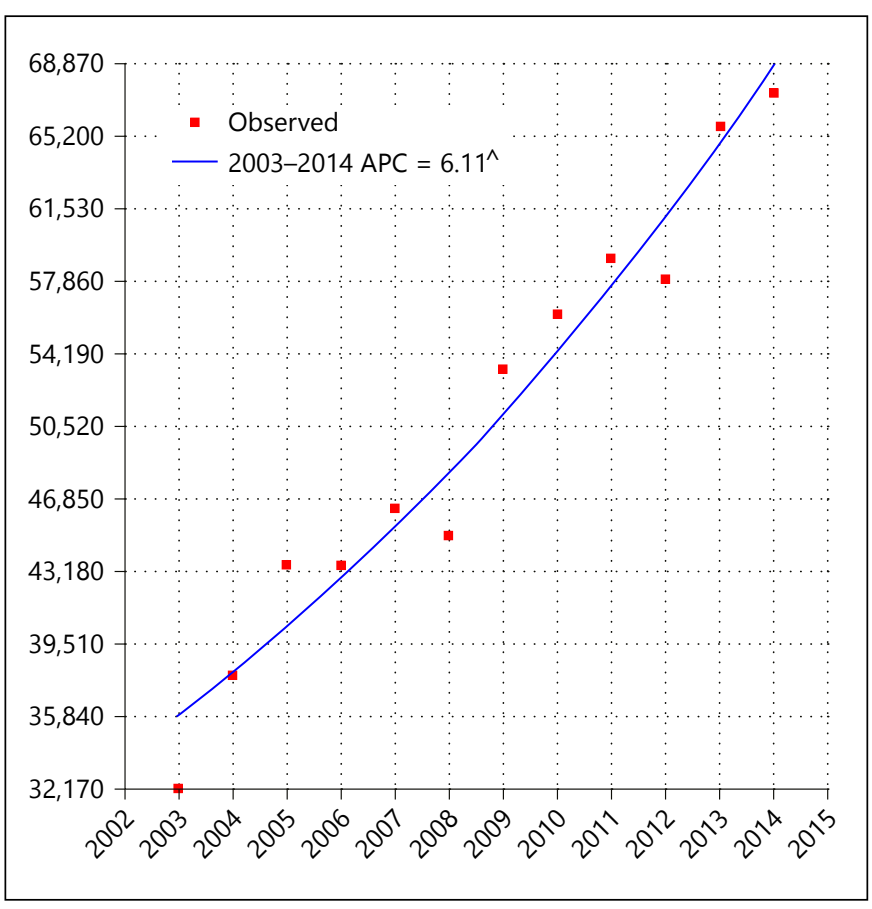

Fig. 1. Trends in the number of first-time cannabis treatment admission in Eu-22 - joinpoint regression outcome, 2003-2014. APC, annual percent change - observed.

use in the general population could have a noticeable impact on the number of people entering drug treatment. In general, cannabis treatment demands will primarily arise from intensive cannabis users $[5,19,20]$, as these are at a greater risk of developing problems with their drug use. This analysis highlighted a 20,000 increase in new entrants to cannabis treatment between 2003 and 2014 who reported daily cannabis use [2, 7, $21]$. In addition, the increased time lag for cannabis clients between start of cannabis use and demand for treatment identified here (from 6 years in 2003 to 8 years in 2014) implies longer (pre-treatment) drug use careers, and increased potential risk of developing cannabis-related problems [22].

Changes in perceived risks related to cannabis use may also play a role in treatment seeking behaviour. According to the 2014 Eurobarometer survey, an increase in risk perception for cannabis use was reported among young people aged 15-20 years. Although lower than for other drugs - such as heroin or cocaine - its increase may reflect a more general increase in the perceived need for cannabis treatment [23].

Recent changes in the cannabis market may also have some indirect influence on the increasing trends witnessed in cannabis treatment demands, although at different speed and timing compared to changes in prevalence. In recent years, for example, an increase in cannabis potency (delta-9-tetrahydrocannabinol [THC] concentrations in cannabis) identified for both herbal cannabis and cannabis resin in some European countries [5], may have led to stronger acute effects and more negative consequences in the short and long term for cannabis users $[24,25]$. In particular, the regular use of cannabis with a higher THC content and a lower CBD concentration may increase the risk of psychological problems and potentially increase the pool of cannabis users who might be in need of treatment [5, 26].

The different timing of changes in prevalence and market may lead to 2 possible hypotheses: the first concerns recent changes in drug market, which may have accentuated problems of an existing group of population at risk and the second hypothesis is that a new group of consumers may have started using higher potency cannabis later developing cannabis-related problems faster; this new group could have added up to the first group causing an increase in the number of those demanding cannabis treatment. It is necessary to further investigate to understand the different timing in prevalence and market and further explore those hypotheses.

It can also be argued that in some countries an increase in the offer or availability of treatment services targeting cannabis-specific problems may have influenced the rise in registered treatment demands for this drug [2, $10]$. While in the past people with opioid dependence were the primary target group for drug treatment services, the number of heroin users seeking treatment has been on the decline for some years and problem cannabis users have in some cases become a new focus for health care systems.

Changes in drug policy may also impact cannabis treatment demands, in particular impact changes in referrals to treatment. Two differing trends in policy approaches can be identified in European countries, both of which can potentially lead to an increase in treatment demands. First, there has been a tendency towards a less punitive approach in cannabis policy in several European countries, which may have contributed to an increase in referrals to treatment as alternative to punishment [27]. A recent analysis on relation between political changes and treatment uptake has shown that while the number of clients with primary cannabis problems has generally increased, this increase does not seem to be substantially stronger in the countries that intro- 


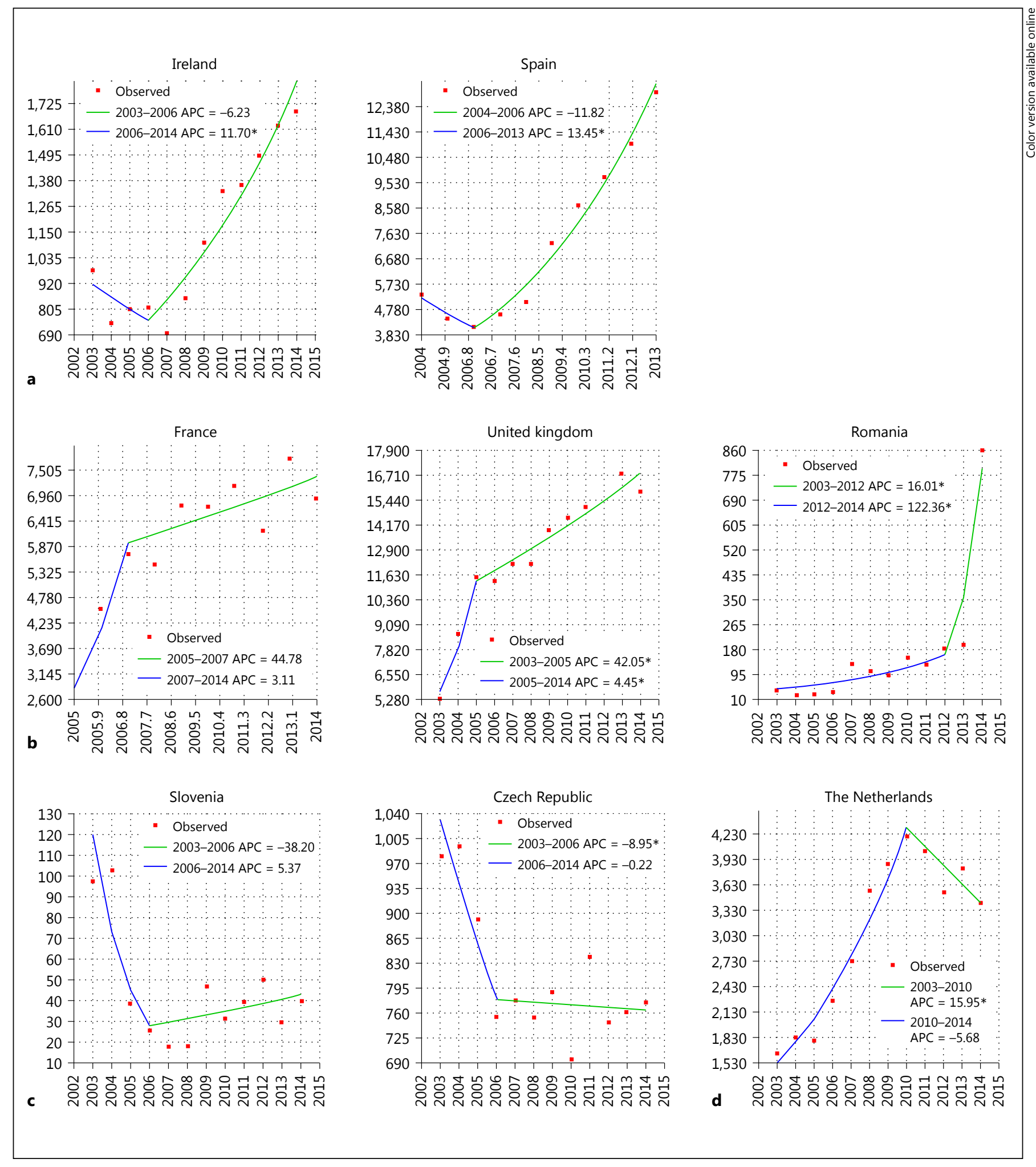

Fig. 2. Joinpoint regression in first-time cannabis treatment admission in countries with broken trends, 2003-2014. a Countries with initial decrease followed by increase. b Countries with 2 peri- ods of increase, where one phase was more accentuated than the other. c Countries with a decrease followed by a stable phase. d Countries with an initial increase followed by decrease. 
duced more permissive cannabis policies [28]. On the other hand, drug policies less tolerant towards cannabis use may also translate in an increased number of cannabis users not being socially tolerated, and therefore referred to drug treatment in order to decrease their use. Overall, however, available data does not show any major changes in the percentage of cannabis clients referred to treatment from the criminal justice system (court, probation and police) in the period covered by this review $[2,3]$.

Finally, since its implementation in the early 2000s, the data collection on drug treatment demand has improved and coverage has increased over the years. The improvement in monitoring, including data reporting and coverage at national level, could mean more treatment episodes now reported. This would likely be the case across drug types and so in absolute terms this should impact trends for other drugs as well as cannabis [2]. A further potential documentation and monitoring factor is linked with the recent increase of new psychoactive substances that have entered Europe's drug market, including a range of synthetic cannabinoid products. These drugs are characterised by high potency and problematic risk profiles, which may have led people to seek for drug treatment [29-31]. There are some reports that the use of synthetic cannabinoids may be registered as the use of cannabis in some treatment entry systems.

Detailed country-level analysis is outside the scope of this paper, but nevertheless, it represents an important starting point for future investigations, especially in view of the country differences related to the trends of cannabis treatment demands.

\section{Limitations}

Estimates included in the paper are based on the best available data at the European level; however, the review identified some clear gaps in existing epidemiological data on cannabis treatment demand. Specifically, only 22 countries provided sufficient data to be included in this analysis and results are therefore not generalizable to Europe as a whole.

It is also important to acknowledge that while treatment data coverage may be reasonable for specialised outpatient and inpatient treatment facilities, the coverage for other treatment providers, for example, general practitioners or other agencies remains low. It is possible that the number of treatments provided by these other parts of the system as well as their proportion might have changed during the period analysed, and this may have had implications for the results of the analysis. Furthermore, data coverage has changed during the period of analysis. During the study period, the European treatment monitoring system has been expanded with the inclusion of additional treatment centers, which may have influenced the overall increase in the number of clients entering drug treatment, although we note that the percentages of cannabis treatment demands have also increased as a percentage of all drugs during this time. In addition, specific information on the types and characteristics of centres that was added to the data-reporting system is not available. Changes in some national monitoring systems (in Italy, data was reported on individual records at the national level since 2010; in Latvia, methodological changes occurred between 2013 and 2014) may have influenced numbers and results. However, as the standards applied and the procedures implemented have remained largely stable during the observation period, it can be assumed that the trend has not been substantially biased by those factors $[8,9]$.

We also acknowledge that treatment monitoring at the EU level is based on the transfer of aggregated data from national sources to the European level, which also limits the possibilities of EMCDDA quality control at the datacollection level. Furthermore, double-counting of cases can only be partly excluded. When the absolute number of individuals is considered, the analysis may be affected by the size of the population entering drug treatment according to the country's population size and the extent of treatment provision for cannabis problems. Therefore, variations within the population in each country could bias the outcomes $[2,3,8]$.

Although data is collected according to the same TDI protocol in all European countries, based on the same 24 items, some differences between countries and within each country in the way data is collected still remain. This could also refer to the main substance, which is the core element of this analysis. This should be considered when interpreting the data. In the TDI protocol, no specific category for "synthetic cannabinoids" exists; this means clients entering drug treatment for the use of these substances are likely to be recorded under the general category "cannabis" and cannot therefore be separated from the current data [8].

Outcomes of the joinpoint regression should be treated with caution when absolute numbers are included because the dependent variable scale is changing according to the absolute count of new clients entering treatment, which could mean that an APC comparison between countries would not be found to be valid. Therefore, any 
comparison between country graphs should be undertaken with caution.

This study did not consider the analysis carried out on the use of other substances (defined in the protocol as "secondary drugs") in combination with cannabis as primary drug (i.e. with cannabis as main reason for people to enter drug treatment, as defined in the TDI Protocol - see Methods and Measures). A specific analysis on polydrug users conducted in 2009 reveals that the lowest proportion of clients who are polydrug users is found among primary cannabis clients compared to users of other drugs entering treatment. Cannabis clients were most commonly using alcohol in combination with cannabis [32].

Finally, it has to be remembered that while treatment provision for cannabis-related problems has increased over the last years in European countries, a clear and harmonised definition regarding the actual treatment and interventions provided for cannabis users is not available at present $[10,33]$.

\section{Conclusions}

This paper has provided evidence of an increase in the overall treatment demand for cannabis-related problems in Europe with differentiated trends evident at the national level. It is apparent that many important questions that are central to the current policy debate remain unanswered [34]. Nevertheless, people with cannabis-related problems represent an increasing proportion of treatment demands in specialised facilities and their needs are significantly different when compared to the needs of opioid clients, which have historically been the focus of public health interventions. National differences and contextual factors remain to be investigated and improv- ing the understanding of the profiles and needs of different sub populations of cannabis clients is an important future research priority. It is important, in particular, to further investigate the differences in trends by groups of countries: firstly comparing the countries with increasing trends and those with decreasing trends; in the second place, among the countries with increasing trends, a comparison has to be made between those with an initial decrease followed by an increase and those with 2 periods of increase, the second comparison being more accentuated than the other. Further investigation is also needed on patterns of drug use of cannabis users and the range of severity of use of other substances among cannabis clients, as less data is available at the national level. Finally, an increased understanding of the reasons for national differences and trends as well as further analysis of possible explanatory factors would be a prerequisite for the development of appropriate and targeted service responses to cannabis related problems in Europe.

\section{Contributors}

All the authors contributed to write the manuscript, concurred with the submission and approved of the final manuscript.

\section{Acknowledgments}

Lucas Wiessing, EMCDDA. Julian Vicente, EMCDDA. National Treatment Demand Experts (REITOX National Focal Points).

\section{Disclosure Statement}

The authors declare that they do not have any conflicts of interest related to the results of the analysis presented in the paper.

\section{References}

1 UNODC: World Drug Report 2016. New York, 2016, p 105.

2 European Monitoring Centre for Drugs and Drug Addiction: European Drug Report 2016: Trends and Developments. Lisbon, EMCDDA, 2016. http://www.emcdda.europa. eu/edr2016.

3 EMCDDA: 2016 Statistical Bulletin, 2016.

4 Hall W, Degenhardt L, Lynskey M: Ageing $\mathrm{CD}$ of $\mathrm{H}$ and: The health and psychological effects of cannabis use. Monogr Ser 2001; 182.

5 WHO: The Health and Social Effects of Nonmedical Cannabis Use. 2016. http://who.int/ substance_abuse/publications/msbcannabis. pdf?ua $=1$.

6 United Nations Office on Drugs and Crimes: Discussion Paper Cannabis A Short Review, 2012, pp 1-29.

7 EMCDDA: A Cannabis Reader: Global Issues and Local Experiences. 2008. http://www. emcdda.europa.eu.

8 EMCDDA: Treatment Demand Indicator Standard Protocol 3.0, 2012.

9 EMCDDA: TDI Methodological Information on 30 European Countries Methods and Definitions of TDI Data Reported to the EMCDDA in 2014, 2014.
10 Schettino J, Leuschner F, Kasten L, Tossman P: Treatment of Cannabis-Related Disorders in Europe. 2015 (http://www.emcdda.europa. $\mathrm{eu} / \mathrm{attachements.cfm})$.

11 Institute NC: Joint Point Regression, 2015.

12 Griffiths P, Mounteney J, Laniel L: Understanding changes in heroin availability in Europe over time: emerging evidence for a slide, a squeeze and a shock. Addiction 2012; 107:1539-1540.

13 Rush B, Urbanoski K: Estimating the demand for treatment for cannabis-related problems in Canada. Int J Ment Health Addict 2007;5: 181-186. 
14 EMCDDA: European Drug Report 2016. Lisbon, EMCDDA, 2016. http://www.emcdda. europa.eu/edr2016.

15 EMCDDA: Annual Report 2005: State of the Drugs Problem in Europe, 2005, p 96.

16 Barrio G, Montanari L, Bravo MJ, Guarita B, de la Fuente L, Pulido J, et al: Trends of heroin use and heroin injection epidemics in Europe: findings from the EMCDDA treatment demand indicator (TDI). J Subst Abuse Treat 2013;45:19-30.

17 Roxburgh A, Hall WD, Degenhardt L, McLar en J, Black E, Copeland J, et al: The epidemiology of cannabis use and cannabis-related harm in Australia 1993-2007. Addiction 2010;105:1071-1079.

18 Sánchez-Niubò A, Sordo L, Fortiana J, Brugal MT, Domingo-Salvany A: Incidence trends of cannabis and cocaine use from periodic Spanish general population surveys: effect of standardizing results by age structure. Addiction 2013;108:1450-1458.

19 Spanish National Focal: 2011 Spanish National Report. 2011. http://www.emcdda.europa. eu/html.cfm/index191736EN.html.

20 Kaar SJ, Gao CX, Lloyd B, Smith K, Lubman DI: Trends in cannabis-related ambulance presentations from 2000 to 2013 in Melbourne, Australia. Drug Alcohol Depend 2015; 155:24-30.
21 Di Forti M, Sallis H, Allegri F, Trotta A, Ferraro L, Stilo SA, et al: Daily use, especially of high-potency cannabis, drives the earlier onset of psychosis in cannabis users. Schizophr Bull 2014;40:1509-1517.

22 Monshouwer K, Smit F, De Graaf R, van Os J, Vollebergh W: First cannabis use: does onset shift to younger ages? Findings from 1988 to 2003 from the Dutch National School Survey on Substance Use. Addiction 2005;100:963-970.

23 European Commission Directorate-General for General Communication: Flash Eurobarometer 401, Young People and Drugs Report, 2014, pp 1-162.

24 Toxicodependência OE da D e: Cannabis production and markets in Europe. Obs Eur da Drog eToxicodependência 2012;274.

25 King L: Understanding Cannabis Potency and Monitoring Cannabis Products in Europe, 2008.

26 Niesink RJM, Rigter S, Koeter MW, Brunt TM: Potency trends of $\Delta 9$-tetrahydrocannabinol, cannabidiol and cannabinol in cannabis in the Netherlands: 2005-15. Addiction 2015;110: 1941-1950.
27 EMCDDA: European Monitoring Centre for Drugs and Drug Addiction: Annual Report 2011. The State of the Drugs Problem in Europe, 2011, p 112.

28 Adam C, Raschzok A: Cannabis policy and the uptake of treatment for cannabis-related problems. Drug Alcohol Rev 2017;36:171-177.

29 Winstock AR, Barratt M: Synthetic cannabis: a comparison of patterns of use and effect profile with natural cannabis in a large global sample. Drug Alcohol Depend 2013;131:106-111.

30 Pirona A, Atkinson A, Sumnall H: Health Responses to New Psychoactive Substances, 2016, p 30.

31 Evren C, Bozkurt M: Synthetic cannabinoids: crisis of the decade. Dusunen Adam 2013;26: $1-11$.

32 Montanari L, Guarita B: Polydrug Use among Drug Treatment Clients in Europe. What Implications for Treatment? 20 Years of Monitoring - Key Indicators Expert Working Groups, 2015.

33 Degenhardt L, Hall W: Extent of illicit drug use and dependence, and their contribution to the global burden of disease. Lancet 2012; 379:55-70.

34 Degenhardt L, Stockings E, Patton G, Hall WD, Lynskey M: The increasing global health priority of substance use in young people. Lancet Psychiatry 2016;3:251-264. 